\title{
CORRESPONDENCE
}

\section{Nasu-Hakola disease and primary microglial dysfunction}

Marino M. Bianchin, Kelin C. Martin, Ana C. de Souza, Marina A. de Oliveira and Carlos R. de Mello Rieder

In their article (Microglia in neurodegenerative disease. Nat. Rev. Neurol. 6, 193-201;2010) ${ }^{1}$ Perry et al. provide a comprehensive review of the involvement of microglia in neurodegenerative disease. The authors highlight the substantial interest that exists regarding the possible roles of activated microglia in disease pathogenesis, and question whether activated microglia exacerbate pathology or aid tissue repair in neurodegenerative diseases. In all of the diseases used as examples by the authors, microglial activation is secondary to other pathological processes that affect the CNS. In order to contribute to this discussion, we would like to highlight the interesting example of Nasu-Hakola disease, and discuss how microglial dysfunction might cause substantial brain damage. ${ }^{2-6}$ This example of microglial dysfunction causing brain damage is important because it might offer insights into the importance of microglial homeostasis in the brain.

Nasu-Hakola disease-also known as polycystic lipomembranous osteodysplasia with sclerosing leukoencephalopathy (PLOSL) - is a genetic disorder characterized by progressive presenile dementia and bone cysts. ${ }^{2-7}$ Patients with Nasu-Hakola disease develop a variant of frontotemporal dementia associated with cyst-like bone lesions (Figure 1). The disease usually starts during the $4^{\text {th }}$ decade of life, leading to death after only a few years. ${ }^{2-7}$ In contrast to other diseases, evidence strongly suggests that in Nasu-Hakola disease CNS damage is directly caused by microglial dysfunction. ${ }^{2,3,8,9}$ Histological examination of brains from patients with Nasu-Hakola disease shows that neuronal loss, and astrocyte proliferation and hypertrophy, occurs mainly in the white matter of the frontotemporal lobe and basal ganglia. ${ }^{3,10}$ Nevertheless, the most interesting feature of this disease is a well-documented activation of microglia in the cerebral white matter of patients with this condition, and this prominent microglial activation might be the primary underlying factor responsible for Nasu-Hakola disease. ${ }^{3,11}$

Genetic studies have shown that mutations in the genes TREM2 and TYROBP, which encode the proteins
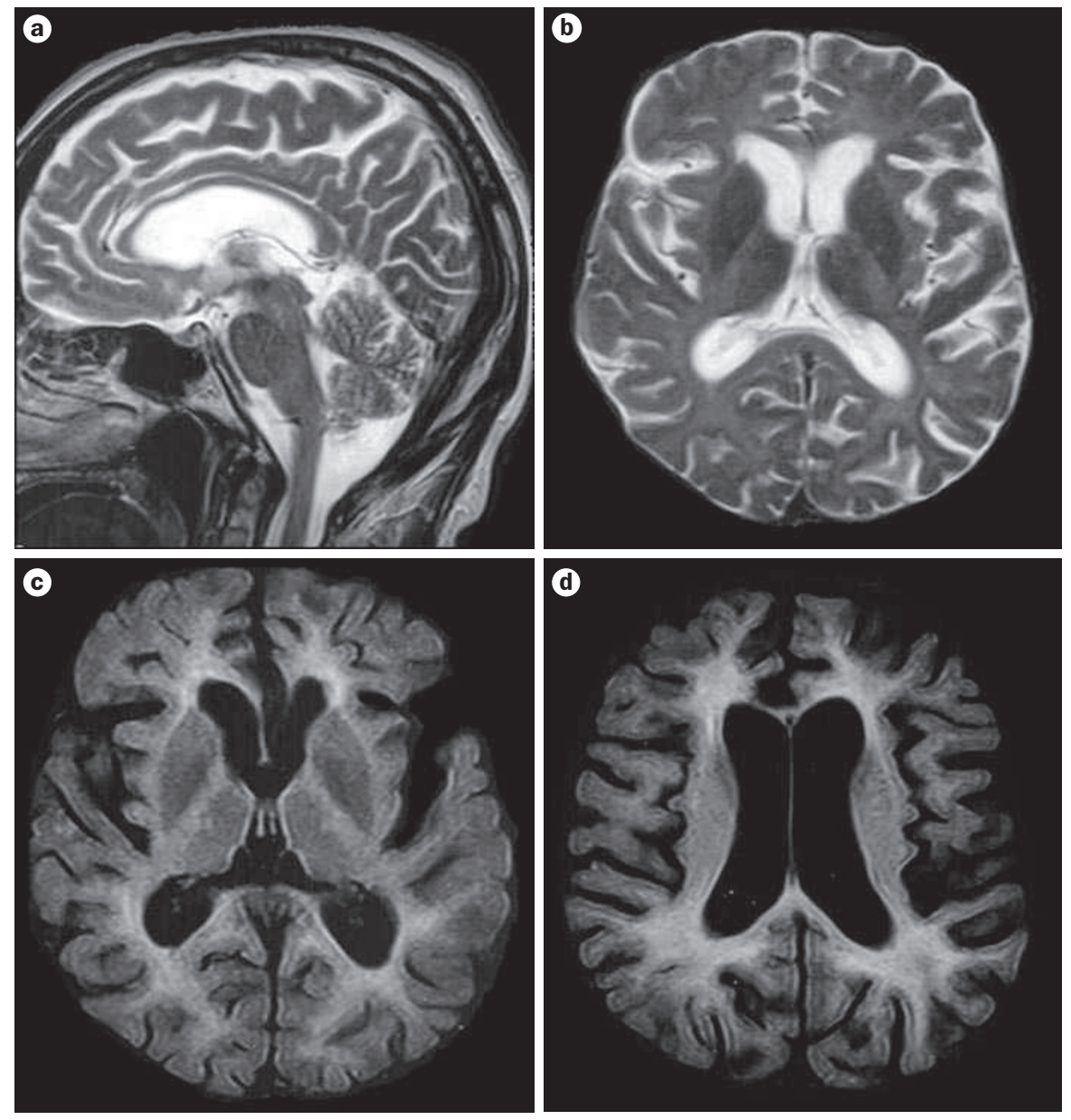

Figure 1 | Brain MRI scans (1.5T) of a patient with clinical, pathological and molecular diagnosis of Nasu-Hakola disease. a | Sagittal and $\mathbf{b}$ | axial T2-weighted images showing marked brain atrophy and abnormal signal in white matter. c,d | Fluid-attenuated inversion recovery sequences revealing brain atrophy and signal abnormalities in white matter and basal ganglia. 
Nasu-Hakola disease as well as other CNS diseases. Although all TREM2TYROBP functions have not been fully elucidated, Nasu-Hakola disease might be an interesting example of how primary microglial dysfunction can damage the CNS.

Nasu-Hakola disease is emerging as the prototype of a primary microglial disorder of the CNS., ${ }^{2,8}$ Whether Nasu-Hakola disease is a unique disorder, or if this condition is the first identified disorder in an entirely new class of diseases that one could eventually call 'microgliopathies', is unknown. Clinical observations of patients with Nasu-Hakola disease and molecular studies on the TREM2TYROBP pathway might offer interesting insights into microglial physiology and the involvement of this cell type in neurodegenerative disorders. We believe that pharmacological modulation of the TREM2-TYROBP pathway might offer new therapeutic strategies for the treatment of neurological diseases.

Division of Neurology, Hospital de Clinicas de Porto Alegre, Ramiro Barcelos 2350, Porto Alegre, RS, Brazil (M. M. Bianchin, K. C. Martin, A C. de Souza, M. A. de Oliveira, C. R. de Mello Rieder).
Correspondence to: M. M. Bianchin mmbianchin@hotmail.com

\section{doi:10.1038/nrneurol.2010.17-c1}

\section{Acknowledgments}

The authors are supported by Brazilian governmental research grants (CNPq, FAPERGS and Propesq/UFRGS).

\section{Competing interests}

The authors declare no competing interests.

1. Perry, V. H., Nicoll, J. A. \& Holmes, C. Microglia in neurodegenerative disease. Nat Rev. Neurol. 6, 193-201 (2010).

2. Bianchin, M. M. et al. Nasu-Hakola disease (polycystic lipomembranous osteodysplasia with sclerosing leukoencephalopathy-PLOSL): a dementia associated with bone cystic lesions. From clinical to genetic and molecular aspects. Cell Mol. Neurobiol. 24, 1-24 (2004).

3. Paloneva, J. et al. CNS manifestations of Nasu-Hakola disease: a frontal dementia with bone cysts. Neurology 56, 1552-1558 (2001)

4. Chouery, E. et al. Mutations in TREM2 lead to pure early-onset dementia without bone cysts. Hum. Mutat. 29, E194-E204 (2008).

5. Paloneva, J. et al. Loss-of-function mutations in TYROBP (DAP12) result in a presenile dementia with bone cysts. Nat. Genet. 25, 357-361 (2000).

6. Paloneva, J. et al. DAP12/TREM2 deficiency results in impaired osteoclast differentiation and osteoporotic features. J. Exp. Med. 198, 669-675 (2003).

7. Bianchin, M. M., Lima, J. E., Natel, J. \& Sakamoto, A. C. The genetic causes of basal ganglia calcification, dementia, and bone cysts: DAP12 and TREM2. Neurology 66, 615-616 (2006).

8. Thrash, J. C., Torbett, B. E. \& Carson, M. J. Developmental regulation of TREM2 and DAP12 expression in the murine CNS: implications for Nasu-Hakola disease. Neurochem. Res. 34, 38-45 (2009).

9. Neumann, H. \& Takahashi, K. Essential role of the microglial triggering receptor expressed on myeloid cells-2 (TREM2) for central nervous tissue immune homeostasis. J. Neuroimmunol. 184, 92-99 (2007).

10. Tanaka, J. Nasu-Hakola disease: a review of its leukoencephalopathic and membranolipodystrophic features. Neuropathology 20 (Suppl.), S25-29 (2000).

11. Kiialainen, A., Hovanes, K., Paloneva, J., Kopra, O. \& Peltonen, L. Dap12 and Trem2, molecules involved in innate immunity and neurodegeneration, are co-expressed in the CNS. Neurobiol. Dis. 18, 314-322 (2005).

12. Paloneva, J. et al. Mutations in two genes encoding different subunits of a receptor signaling complex result in an identical disease phenotype. Am. J. Hum. Genet. 71, 656-662 (2002).

13. Cella, M. et al. Impaired differentiation of osteoclasts in TREM-2-deficient individuals. J. Exp. Med. 198, 645-651 (2003).

14. Colonna, M. TREMs in the immune system and beyond. Nat. Rev. Immunol. 3, 445-453 (2003).

15. Takahashi, K., Rochford, C. D. \& Neumann, H. Clearance of apoptotic neurons without inflammation by microglial triggering receptor expressed on myeloid cells-2. J. Exp. Med. 201 647-657 (2005). 